\title{
Discontinuation of tyrosine kinase inhibitor therapy in chronic myeloid leukaemia (EURO-SKI): a prespecified interim analysis of a prospective, multicentre, non-randomised, trial
}

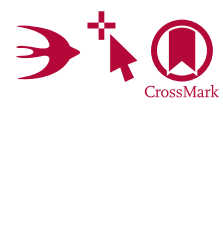

\author{
Susanne Saussele*, Johan Richter*, Joelle Guilhot* , Franz X Gruber, Henrik Hjorth-Hansen, Antonio Almeida, Jeroen JW M Janssen, Jiri Mayer, \\ Perttu Koskenvesa, Panayiotis Panayiotidis, Ulla Olsson-Strömberg, Joaquin Martinez-Lopez, Philippe Rousselot, Hanne Vestergaard, \\ Hans Ehrencrona, Veli Kairisto, Katerina Machová Poláková, Martin C Müller, Satu Mustjoki, Marc G Berger, Alice Fabarius, \\ Wolf-Karsten Hofmann, Andreas Hochhaus, Markus Pfirrmannt, Francois-Xavier Mahont, on behalf of the EURO-SKI investigatorsł
}

\section{Summary}

Background Tyrosine kinase inhibitors (TKIs) have improved the survival of patients with chronic myeloid leukaemia. Many patients have deep molecular responses, a prerequisite for TKI therapy discontinuation. We aimed to define precise conditions for stopping treatment.

Methods In this prospective, non-randomised trial, we enrolled patients with chronic myeloid leukaemia at 61 European centres in 11 countries. Eligible patients had chronic-phase chronic myeloid leukaemia, had received any TKI for at least 3 years (without treatment failure according to European LeukemiaNet [ELN] recommendations), and had a confirmed deep molecular response for at least 1 year. The primary endpoint was molecular relapse-free survival, defined by loss of major molecular response (MMR; >0 1\% BCR-ABL1 on the International Scale) and assessed in all patients with at least one molecular result. Secondary endpoints were a prognostic analysis of factors affecting maintenance of MMR at 6 months in learning and validation samples and the cost impact of stopping TKI therapy. We considered loss of haematological response, progress to accelerated-phase chronic myeloid leukaemia, or blast crisis as serious adverse events. This study presents the results of the prespecified interim analysis, which was done after the 6-month molecular relapse-free survival status was known for 200 patients. The study is ongoing and is registered with ClinicalTrials.gov, number NCT01596114.

Findings Between May 30, 2012, and Dec 3, 2014, we assessed 868 patients with chronic myeloid leukaemia for eligibility, of whom 758 were enrolled. Median follow-up of the 755 patients evaluable for molecular response was 27 months (IQR 21-34). Molecular relapse-free survival for these patients was $61 \%$ (95\% CI 57-64) at 6 months and $50 \%(46-54)$ at 24 months. Of these 755 patients, 371 (49\%) lost MMR after TKI discontinuation, four (1\%) died while in MMR for reasons unrelated to chronic myeloid leukaemia (myocardial infarction, lung cancer, renal cancer, and heart failure), and 13 (2\%) restarted TKI therapy while in MMR. A further six (1\%) patients died in chronic-phase chronic myeloid leukaemia after loss of MMR and re-initiation of TKI therapy for reasons unrelated to chronic myeloid leukaemia, and two (<1\%) patients lost MMR despite restarting TKI therapy. In the prognostic analysis in 405 patients who received imatinib as first-line treatment (learning sample), longer treatment duration (odds ratio [OR] per year 1.14 [95\% CI 1.05-1·23]; $\mathrm{p}=0$-0010) and longer deep molecular response durations $(1 \cdot 13$ [1.04-1.23]; $\mathrm{p}=\mathbf{0} .0032$ ) were associated with increasing probability of MMR maintenance at 6 months. The OR for deep molecular response duration was replicated in the validation sample consisting of 171 patients treated with any TKI as first-line treatment, although the association was not significant $(1 \cdot 13[0 \cdot 98-1 \cdot 29] ; \mathrm{p}=0 \cdot 08)$. TKI discontinuation was associated with substantial cost savings (an estimated $€ 22$ million). No serious adverse events were reported.

Interpretation Patients with chronic myeloid leukaemia who have achieved deep molecular responses have good molecular relapse-free survival. Such patients should be considered for TKI discontinuation, particularly those who have been in deep molecular response for a long time. Stopping treatment could spare patients from treatmentinduced side-effects and reduce health expenditure.

Funding ELN Foundation and France National Cancer Institute.

Copyright (C) 2018 Elsevier Ltd. All rights reserved.

\section{Introduction}

Chronic myeloid leukaemia is a model disease for targeted therapy, characterised by the Philadelphia chromosome and its molecular counterpart, the $B C R-A B L 1$ fusion gene. ${ }^{1}$ This specific leukaemic marker coding for a protein tyrosine kinase is considered the driver of the leukaemic process. This discovery led to the development of BCR-ABL1 tyrosine kinase inhibitors (TKIs), which improved the outcomes of patients with chronic myeloid leukaemia. ${ }^{2,3}$ The life expectancy of
Lancet Oncol 2018

Published Online May 4, 2018 http://dx.doi.org/10.1016/ S1470-2045(18)30192-X

See Online/Comment http://dx.doi.org/10.1016/ S1470-2045(18)30206-7

*Joint first authors

†Contributed equally ¥Investigators listed in the appendix (pp 3-6)

Department of Haematology and Oncology, University Hospital Mannheim, Heidelberg University, Mannheim, Germany (S Saussele MD, A Fabarius PhD Prof W-K Hofmann MD); Department of Haematology, Oncology and Radiation Physics, Skåne University Hospital, Lund, Sweden (Prof J Richter MD); Inserm Centre d'Investigation Clinique 1402, Centre Hospitalier Universitaire (CHU) de Poitiers, Poitiers, France (J Guilhot PhD); Department of Haematology, University Hospital of North Norway, Tromsø, Norway (FX Gruber MD); Department of Haematology, St Olavs Hospital, Trondheim, Norway (H Hjorth-Hansen MD); Instituto Portugues de Oncologia de Lisboa de Francisco Gentil, Lisbon, Portugal (A Almeida MD); Department of Haematology, VU University Medical Center Amsterdam, Netherlands (J JW M Janssen MD); Department of Interna Medicine, Haematology and Oncology, Masaryk University and University Hospital Brno Brno, Czech Republic (Prof J Mayer MD); Haematology Research Unit Helsinki (P Koskenvesa MD, Prof S Mustjoki MD) and 


\begin{abstract}
Department of Clinical Chemistry and Haematology (Prof S Mustjoki), University of Helsinki, Helsinki, Finland; Helsinki University Hospital Comprehensive Cancer Center, Helsinki, Finland (P Koskenvesa, Prof S Mustjoki); First Department of Internal Medicine, Laikon General Hospital, National and Kapodistrian University of Athens, Athens, Greece (P Panayiotidis MD); Department of Medical Science and Division of Haematology, University Hospital, Uppsala, Sweden

(U Olsson-Strömberg MD); Hospital Universitario 12 de Octubre, Centro Nacional de Investigaciones Oncológicas,

Centro de Investigación Biomédica en Red de Cáncer, Universidad Complutense de

Research in context

Evidence before this study

We searched PubMed between Jan 1, 2004, and Dec 31, 2011, using the search term "stopping treatment in chronic myeloid leukemia" for English-language articles investigating imatinib discontinuation (the first tyrosine kinase inhibitor [TKI] used in chronic myeloid leukaemia). We identified additional relevant articles by examining the references of the articles identified in the literature search. Aside from several case reports and small pilot studies, only one prospective trial, the STIM trial, was published during that period. In that trial, $61 \%$ of patients had molecular recurrence after discontinuation of TKI treatment, mostly within 6 months. All patients who relapsed responded when imatinib was reintroduced as treatment. Prognostic factors in a multivariable analysis were TKI treatment duration and risk score at time of diagnosis. New definitions for molecular response were introduced in 2012. No other published studies using these new definitions had been published at the time of design of the EURO-SKI trial.
\end{abstract} Madrid, Madrid, Spain

(Prof) Martinez-Lopez MD); Department of Haematology and Oncology, Centre Hospitalier de Versailles, Inserm Unité Mixte de Recherche 1173, Université Versailles

Saint-Quentin-en-Yvelines, Université Paris Saclay, Le Chesnay, France (Prof P Rousselot MD);

Department of Haematology,

Odense University Hospital, Odense, Denmark (H Vestergaard MD);

Department of Clinical Genetics

and Pathology, Laboratory

Medicine, Office for Medical Services, Lund, Sweden (H Ehrencrona MD); Division of Clinical Genetics, Lund

University, Lund, Sweden (H Ehrencrona); Department of Clinical Chemistry and

Department of Genetics, Turku

University Central Hospital,

Turku, Finland (V Kairisto MD);

Institute of Haematology and

Blood Transfusion, Prague, Czech Republic

(K Machová Poláková PhD);

Institute for Hematology and Oncology, Mannheim,

Germany (M C Müller MD);

Hématologie Biologique and Equipe d'Accueil 7453

Hemopaties Chroniques:

Heterogeneite Intra-clonale,

Microenvironnement et

Resistance Therapeutique, $\mathrm{CHU}$

Estaing and Université

Clermont Auvergne,

Clermont-Ferrand, France

(Prof M G Berger MD); Klinik für Innere Medizin II,

\section{Added value of this study}

In this multicentre, prospective study using standardised monitoring, we confirmed that patients with chronic myeloid

patients with chronic myeloid leukaemia receiving TKI treatment is similar to that of the general population. ${ }^{4}$ A substantial proportion of patients with chronic-phase chronic myeloid leukaemia achieve a deep molecular response on TKI therapy, which is defined by $B C R-A B L 1$ transcript levels measured with real-time (rt)PCR., ${ }^{5,6}$

The proof of concept for stopping TKI treatment came from the STIM1 trial, 7 in which the criterion for stopping imatinib was undetectable $B C R$ - $A B L 1$ transcript for 2 years. Nearly $40 \%$ of patients in that trial were still in molecular remission (defined as a negative PCR result) after 3 years of imatinib discontinuation. ${ }^{7}$ The A-STIM study ${ }^{8}$ validated the loss of major molecular response (MMR) as a criterion for restarting TKI therapy. Other studies ${ }^{9,10}$ also confirmed the possibility of stopping TKI safely and successfully after therapy with second-generation TKIs, such as dasatinib and nilotinib. However, the exact conditions for stopping treatment are not yet defined because of small patient samples. As a result, some of the existing clinical treatment recommendations have restricted TKI discontinuation to the clinical trial setting.

To our knowledge, the European Stop Kinase Inhibitor (EURO-SKI) trial is the most extensive investigation of TKI discontinuation in a setting of decentralised, but standardised, molecular monitoring. We aimed to define the precise conditions for stopping treatment in patients with chronic myeloid leukaemia in deep molecular response.

\section{Methods}

\section{Study design and participants}

For this prospective, single-arm, open-label, nonrandomised trial, we enrolled patients with chronic leukaemia have good molecular relapse-free survival. To our knowledge, this study is the first to show that more exact criteria for stopping treatment could be defined using prognostic modelling to predict treatment-free remission at 6 months in a large cohort of patients. In multivariable analysis, treatment duration, interferon pre-treatment, and deep molecular response duration were significantly associated with the success of imatinib cessation. Deep molecular response duration had the largest effect on the success of treatment cessation, with a yearly increase of roughly $3 \%$ in the probability of staying in major molecular remission at 6 months over the observed time period. Considering only the drug price (before introduction of generic imatinib), discontinuation of imatinib therapy saved $€ 22$ million.

\section{Implications of all the available evidence}

Our results should help physicians in selecting patients with chronic myeloid leukaemia who are eligible for treatment discontinuation, and should be included in recommendations and guidelines on TKI discontinuation. Additionally, increasing the number of patients with chronic myeloid leukaemia who are recommended for TKI treatment discontinuation could have a large economic impact.

myeloid leukaemia at 61 European centres in 11 countries (appendix p 7). Eligible patients were aged 18 years or older, had BCR-ABL1-positive chronic-phase chronic myeloid leukaemia, and were receiving first-line or second-line (because of toxicity with first-line TKI) treatment with any TKI or taking a TKI as part of a combination treatment. The minimum treatment duration with a TKI at any dose was 3 years, and the minimum duration of deep molecular response was 1 year. Deep molecular response was defined as detectable $B C R-A B L 1 \quad(\leq 0 \cdot 01 \%$ on the International Scale $^{12}$ ) or undetectable $B C R-A B L 1$ in samples with 10000 or more $A B L 1$ transcripts or 24000 or more GUS transcripts. To be included, patients had to have at least three PCR results showing deep molecular response within the year (give or take 2 months) before study entry and no results greater than $0 \cdot 01 \% B C R-A B L$ during the same period. The date of the first recording of a deep molecular response and the dates of the last three recordings of deep molecular response before TKI discontinuation were mandatory.

We excluded patients with previous allogeneic stemcell transplantation, TKI failure (according to European LeukemiaNet [ELN] recommendations), ${ }^{13}$ or an active concomitant malignancy. A typical $B C R-A B L 1$ transcript (majority e13a2 or e14a2) was necessary. Pre-treatment with interferon alfa, cytarabine, or hydroxycarbamide was allowed. Fertile women were included if they used an effective contraceptive. Additionally, patients had to have records on the date of diagnosis and values for the prognostic factors in the Sokal, Euro, and European Treatment and Outcome Study (EUTOS) scores (ie, age, 
palpable spleen size enlargement in $\mathrm{cm}$ below the costal margin, platelet count, and percentages of blasts, basophils, and eosinophils in peripheral blood). There were no restrictions on Eastern Cooperative Oncology Group performance status or laboratory findings.

In agreement with each country's public health code, the ethics committee at each centre or State Medical Chamber (for centres in Germany) approved the protocol. All patients were included after written informed consent was obtained. The full protocol of this trial is included in the appendix (pp 10-66).

\section{Procedures}

Molecular response was assessed with rtPCR at designated standardised EUTOS laboratories (each centre was assigned to one laboratory) and reported as the ratio of $B C R-A B L 1$ to $A B L 1$ or GUS on the International Scale. Molecular response assessment followed ELN recommendations for $B C R-A B L 1$ quantification and scoring of deep molecular response, ${ }^{12,14}$ and was done once a month during the first 6 months after TKI discontinuation, every 6 weeks until month 12 , and then every 3 months for at least 3 years. Patients with a confirmed deep molecular response could stop TKI treatment immediately. Molecular recurrence was defined as loss of MMR, corresponding to expression of more than $0 \cdot 1 \% B C R-A B L 1$ transcripts at any time. For patients with loss of MMR, TKI treatment was restarted, ideally with the same TKI. Particularly for the primary endpoint, molecular results were centrally reviewed by members of the study steering committee.

\section{Outcomes}

The primary endpoint was molecular relapse-free survival, which was measured from the date of TKI discontinuation to the date of the first event (loss of MMR or death) or censoring. Observation time was censored on the date of re-initiation of therapy in patients who did not have molecular recurrence or on the date of the last molecular examination in all other patients without an event.

We also assessed molecular relapse-free and treatmentfree survival as an unspecified, post-hoc endpoint. This endpoint differed from the primary endpoint in that restarting therapy without previous molecular recurrence was not censored, but was considered as an event. We decided to assess this endpoint because a patient might have restarted therapy before loss of MMR if they were perceived to be at a high risk of MMR loss or other events linked to withdrawal syndrome; this censoring could be considered as informative.

We assessed factors affecting MMR maintenance at 6 months (prognostic analysis) and the cost impact of TKI discontinuation as secondary endpoints. Adverse events were assessed at each follow-up visit with the Common Toxicity Criteria. Serious adverse events were defined as loss of MMR, complete cytogenetic remission $(>1 \%$ BCR-ABL1), or complete haematological remission (white blood cells $<10 \times 10^{9} / \mathrm{L}$, platelets $<450 \times 10^{9} / \mathrm{L}$, and disappearance of immature circulating cells and all signs and symptoms of the disease), or progression to accelerated-phase chronic myeloid leukaemia or blast crisis, all of which indicated progression of disease. In cases of death, the association of death with chronic myeloid leukaemia was assessed.

This Article presents the results of the prespecified interim analysis, which was planned for after the 6-month molecular relapse-free survival status was known for 200 patients. None of these patients had to restart TKI treatment before molecular relapse. The secondary endpoints of overall survival, progression-free survival, patient-reported quality of life, and symptom burden over time will be reported in the final analysis, which is planned 3 years after the last patient was registered.

\section{Statistical analyses}

On the basis of previous results, ${ }^{15}$ we expected a 6-month molecular relapse-free survival of $40 \%$ or higher. Thus, the prespecified null hypothesis was 6-month molecular relapse-free survival of $40 \%$ or lower. If, at the interim analysis, at least $95(47.5 \%)$ of 200 patients were still in MMR or better (ie, if the lower limit of the two-sided $95 \% \mathrm{CI}$ around $47.5 \%$ [40.4\%] was above $40 \%$ ), the null hypothesis would be discarded at the one-sided significance level of 0.025 (type I error). With 200 patients, a true molecular-relapse free survival of at least $51 \%$ would give more than $80 \%$ power to reject the null hypothesis.

At the final analysis, the null hypothesis of the 3-year molecular relapse-free survival of being $35 \%$ or lower will be tested. If at least 197 (39.4\%) of 500 patients are still in MMR or better, the lower confidence limit of the two-sided $95 \%$ CI around $39.4 \%$ will be higher than $35 \%$ and the null hypothesis will be discarded at the onesided significance level of $0 \cdot 025$. The power to reject the null hypothesis will be higher than $80 \%$ if the true molecular relapse-free survival is at least $42 \%$. A sample size of 500 patients would also allow investigation of about 25 potential prognostic factors, assuming that half of the patients lose their MMR status (relapse) during the 3 years of follow-up and applying the rough estimate that ten events per investigated factor are needed. Although we had planned to recruit 500 patients, many investigators and patients were interested in treatment discontinuation and on registering their data in the trial database to support research. Thus, more patients were recruited than planned.

We estimated molecular relapse-free survival and molecular relapse-free and treatment-free survival using the Kaplan-Meier method. We calculated the cumulative incidence of molecular recurrence by considering restarting treatment without evidence of molecular recurrence and death before molecular recurrence as competing events. ${ }^{16}$

We included all patients with follow-up data in the primary and safety analyses. We considered a patient to
Universitätsklinikum Jena, Jena, Germany (Prof A Hochhaus MD); Institut für Medizinische Informationsverarbeitung, Biometrie und Epidemiologie, Ludwig-MaximiliansUniversität, Munich, Germany (M Pfirrmann PhD); and Bergonié Cancer Institute, Inserm Unit 916, University of Bordeaux, Bordeaux, France (Prof F-X Mahon MD)

Dr Franz X Gruber died in February, 2016

Correspondence to: Prof François-Xavier Mahon, Institut Bergonié, Bordeaux Cedex 33076, France francois-xavier. mahon@u-bordeaux.fr See Online for appendix 


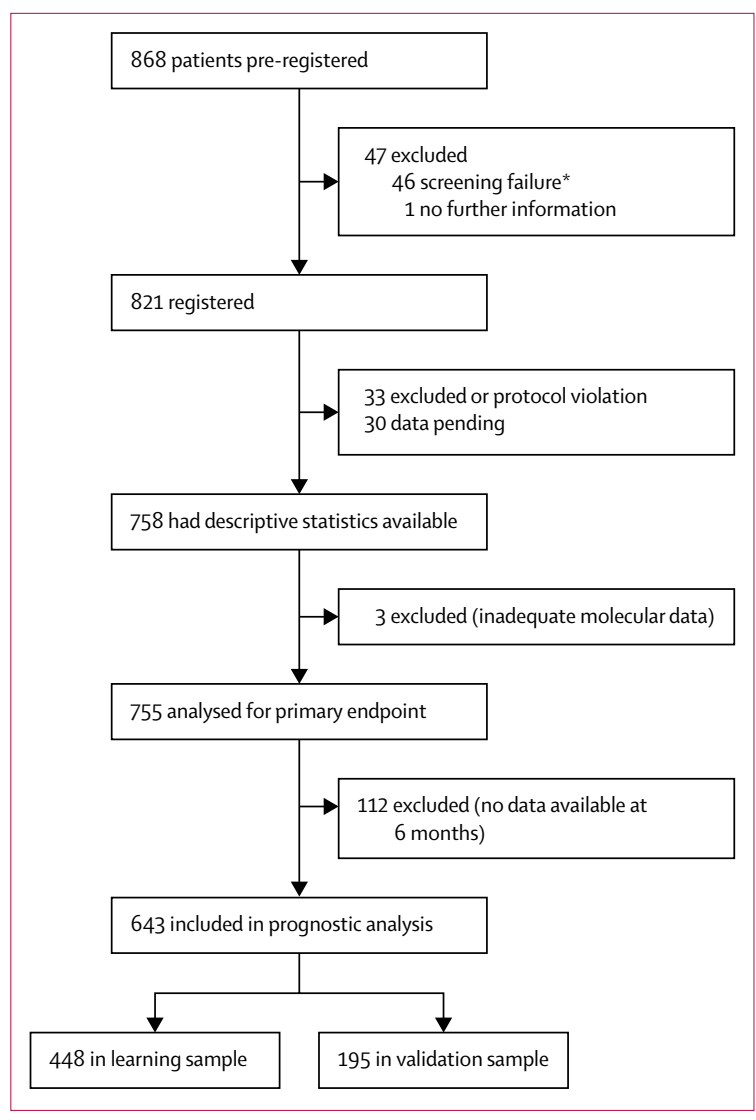

Figure 1: Trial profile

*Deep molecular response not confirmed or other inclusion criteria no longer satisfied.

have been followed up if they had least one molecular result after treatment discontinuation. For all patients, a molecular follow-up of at least 6 months was aimed for, but a minimum follow-up was not required.

We used logistic regression analysis to investigate factors affecting MMR maintenance at 6 months. The learning sample in this analysis included all patients treated with first-line imatinib who had complete data on molecular relapse-free survival status at 6 months and all potential prognostic factors. Candidate prognostic factors were age at diagnosis and TKI discontinuation; sex; Sokal, Euro, EUTOS, and EUTOS long-term survival (ELTS) risk scores (or any variable part of any of those scores); previous interferon treatment; duration of TKI treatment; time to deep molecular response while receiving TKI treatment; and deep molecular response duration before TKI discontinuation. Values of all candidate variables had to be known at the date of TKI discontinuation. These variables were selected either because they were significantly associated with molecular relapse-free survival in previous studies $^{15}$ or because they were suspected to be associated and had been documented in at least $90 \%$ of cases.

To investigate whether the best fit of a continuous variable in the logistic regression model was a straight line, fractional polynomials up to the second degree were considered as alternatives and investigated via a closedtest procedure, as suggested by Royston and Sauerbrei. ${ }^{17}$ The same principles were applied to the multiple regression analysis. Backward elimination was combined with an adaptive algorithm, which selected the best polynomial transformation for each continuous variable in turn to identify independent prognostic factors. Bootstrap resampling was done to assess the stability of the model. ${ }^{18}$ For the final model, the Hosmer and Lemeshow goodness-of-fit test was calculated. ${ }^{19}$ To aid in medical decision making, the linear predictor of the final model was intended to be categorised into risk groups. The classification was done with the minimum $p$ value approach, with adjustment for multiple testing ${ }^{20}$ and assuming that the smallest group could contain at least $20 \%$ of patients. Bootstrap resampling was applied to assess the stability of classification. ${ }^{18,20}$ The predictive quality of the classification was assessed in terms of sensitivity, specificity, and predictive values

Before doing multiple regression analysis, the minimum $\mathrm{p}$ value approach was applied to interferon pre-treatment duration. The reason for this post-hoc analysis was that inclusion of patients with a longer period of interferon pre-treatment could constitute positive selection of patients who were able to stay in chronic phase also without TKI.

Prognostic results were validated in an independent sample. The independent validation sample consisted of all patients who had not been qualified for the learning sample because either their first-line TKI had not been imatinib or they did not have complete values for all candidate variables. After the identification of the final model in the learning sample, the prognostic quality of the same model was examined in the validation sample.

The cost analysis was done for patients pre-treated with imatinib. Months without TKI treatment were counted for each country separately. The assessment of the cost impact of TKI discontinuation included only patients who stopped imatinib. The analysis was done before generic approval in European countries. We accounted for differences in price between countries, and the mean cost of imatinib per month was used to estimate the saving per month for each patient.

Apart from the minimum $\mathrm{p}$ value approach, the significance level of the two-sided p values was 0.05 for all statistical tests. ${ }^{17}$ Analyses were done with SAS version 9.4, an SAS macro for multiple fractional polynomials, or RStudio version 1.0.136.

The study was registered with ClinicalTrials.gov, number NCT01596114.

\section{Role of the funding source}

The funders had no role in study design, data collection, data analysis, data interpretation, or writing of this Article. The corresponding author had full access to all of 
the data and the final responsibility to submit for publication.

\section{Results}

Between May 30, 2012, and Dec 3, 2014, we screened 868 patients with chronic-phase chronic myeloid leukaemia at 61 European centres in 11 countries for eligibility (figure 1). 46 patients were excluded after screening and no further information was available for one patient. Thus, 821 patients were enrolled in the study. Descriptive characteristics were only available for 758 patients at the time of the analysis (the cutoff date for data was Oct 11, 2016) because of exclusion criteria, and molecular analysis was only done for 755 patients because three patients had inadequate molecular data (figure 1).

Baseline characteristics and treatment information for 758 patients is shown in table 1.361 (48\%) of 758 patients had previous treatment with hydroxycarbamide, interferon alfa, or both before TKI cessation. Of the 362 patients who did not have pre-treatment, 338 received imatinib, six received dasatinib, and 18 received nilotinib as first-line TKI therapy. 66 (9\%) of 758 patients had TKIs as part of a combination treatment: nine (1\%) patients received imatinib in combination with anagrelide or hydroxycarbamide, 31 (4\%) received imatinib and interferon alfa, $25(3 \%)$ received imatinib and cytarabine, and one $(<1 \%)$ received imatinib and pioglitazone.

123 (62\% [95\% CI 54-68]) of the first 200 evaluable patients were in MMR or better at 6 months, allowing the null hypothesis of $40 \%$ or lower molecular relapsefree survival at 6 months to be rejected $(p<0 \cdot 0001)$.

Median follow-up of the 755 patients evaluable for molecular response was 27 months (IQR 21-34). 373 (49\%) patients lost MMR (two after restarting TKI therapy while in MMR), and the cumulative incidence of molecular recurrence was $39 \%$ (95\% CI 35-42) at 6 months (figure 2) and 49\% (45-52) at 24 months. Loss of MMR occurred most frequently in the first 6 months after TKI discontinuation (in 297 [80\%] of 373 patients), leading to a molecular relapse-free survival of $61 \%$ (95\% CI 57-64) at 6 months (figure 3) and 50\% (46-54) at 24 months. Of these 755 patients, 371 (49\%) lost MMR after TKI discontinuation, four (1\%) died while in MMR for reasons unrelated to chronic myeloid leukaemia, and $13(2 \%)$ restarted TKI therapy while in MMR. When the 13 TKI restarts before loss of MMR were also counted as an event, molecular relapse-free and treatment-freesurvival was $60 \%(56-63)$ at 6 months and $49 \%(45-52)$ at 24 months (figure 3 ).

448 patients treated with imatinib qualified for prognostic modelling and were included in the learning sample. In this sample, duration of treatment with imatinib, deep molecular response duration, and interferon pre-treatment were significantly associated with MMR maintenance at 6 months after imatinib discontinuation (table 2).

\begin{tabular}{|c|c|}
\hline & Patients $(n=758)$ \\
\hline \multicolumn{2}{|l|}{ Sex } \\
\hline Female & $362(48 \%)$ \\
\hline Male & $396(52 \%)$ \\
\hline Age at diagnosis (years) & $52(41-60)$ \\
\hline Age at TKI discontinuation (years) & $60(50-68)$ \\
\hline Time from diagnosis to TKI discontinuation (years) & $7 \cdot 7(5 \cdot 1-10 \cdot 4)$ \\
\hline Duration of TKI therapy, years & $7 \cdot 5(5 \cdot 0-9 \cdot 9)$ \\
\hline Duration of DMR before TKI discontinuation (years) & $4 \cdot 7(2 \cdot 9-6 \cdot 9)$ \\
\hline \multicolumn{2}{|l|}{ Sokal score* } \\
\hline Low & $259 / 584(44 \%)$ \\
\hline Intermediate & $197 / 584(34 \%)$ \\
\hline High & $128 / 584(22 \%)$ \\
\hline \multicolumn{2}{|l|}{ Euro score† } \\
\hline Low & 239/547 (44\%) \\
\hline Intermediate & $256 / 547(47 \%)$ \\
\hline High & $52 / 547(10 \%)$ \\
\hline \multicolumn{2}{|l|}{ EUTOS score $\ddagger$} \\
\hline Low & $536 / 588(91 \%)$ \\
\hline High & $52 / 588(9 \%)$ \\
\hline \multicolumn{2}{|l|}{ EUTOS long-term survival score* } \\
\hline Low & $408 / 584(70 \%)$ \\
\hline Intermediate & $141 / 584(24 \%)$ \\
\hline High & $35 / 584(6 \%)$ \\
\hline Treatment before TKI & $396(52 \%)$ \\
\hline Hydroxycarbamide & $273(36 \%)$ \\
\hline Hydroxycarbamide and interferon alfa & $66(9 \%)$ \\
\hline Interferon alfa & $22(3 \%)$ \\
\hline Other & $20(3 \%)$ \\
\hline Unknown & $15(2 \%)$ \\
\hline \multicolumn{2}{|l|}{ First-line TKI } \\
\hline Imatinib & $710(94 \%)$ \\
\hline Nilotinib & $33(4 \%)$ \\
\hline Dasatinib & $14(2 \%)$ \\
\hline Unknown & $1(<1 \%)$ \\
\hline \multicolumn{2}{|l|}{ Second-line TKI } \\
\hline Imatinib & $7 / 116(6 \%)$ \\
\hline Nilotinib & $47 / 116(41 \%)$ \\
\hline Dasatinib & $62 / 116(53 \%)$ \\
\hline \multicolumn{2}{|c|}{$\begin{array}{l}\text { Data are } n(\%), n / N(\%) \text {, or median (IQR). The denominator is } 758 \text {, unless } \\
\text { otherwise stated. TKI=tyrosine kinase inhibitor. DMR=deep molecular response. } \\
\text { EUTOS=European Treatment and Outcome Study. *Data were missing for } \\
174 \text { patients. †Data were missing for } 211 \text { patients. } \neq \text { Data were missing for } \\
170 \text { patients. }\end{array}$} \\
\hline
\end{tabular}

Patients pre-treated with interferon for 1.5 years or less were less likely than patients pre-treated for more than 1.5 years to be in MMR 6 months after TKI discontinuation: molecular relapse-free survival at 6 months was $86 \%$ (95\% CI 72-95) in 43 patients who were pretreated for more than 1.5 years and $56 \%(51-60)$ in 405 patients pre-treated for 1.5 years or less (OR 4.93 [95\% CI 2.04-11.94]; $\left.\mathrm{p}_{\text {adiusted }}=0 \cdot 00051\right)$. The probability of maintaining MMR at 6 months did not differ significantly 


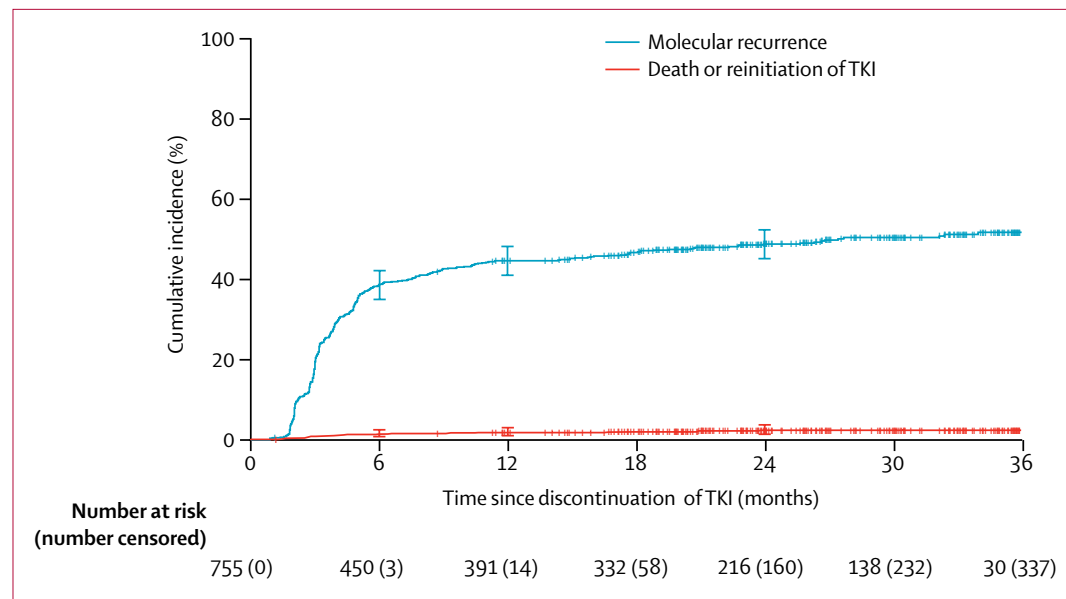

Figure 2: Cumulative incidence of molecular recurrence after TKI discontinuation Bars at 6,12, and 24 months indicate the upper and lower limits of the $95 \% \mathrm{Cls}$ for the estimated incidences. TKI=tyrosine kinase inhibitor.

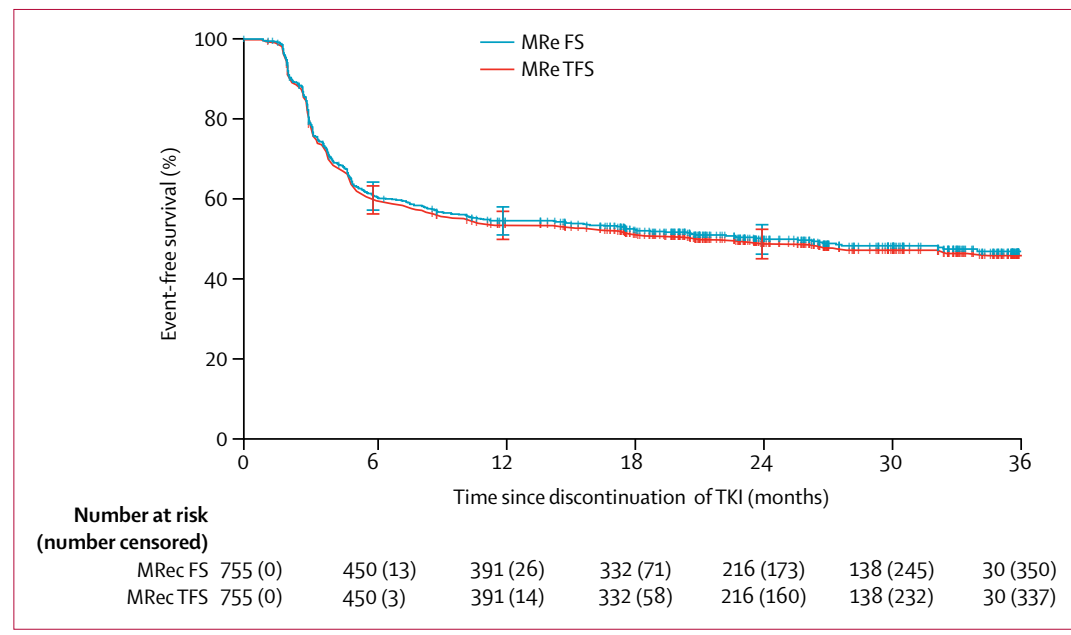

Figure 3: MReFS and MReTFS after TKI discontinuation

Bars at 6, 12, and 24 months indicate the upper and lower limits of the $95 \% \mathrm{Cls}$. MReFS=molecular relapse-free survival. MReTFS=molecular relapse-free and treatment-free survival. TKI=tyrosine kinase inhibitor.

between patients who were not pre-treated with interferon and those who were pre-treated for 1.5 years or less $(1.32$ $[0 \cdot 64-2 \cdot 72] ; p=0 \cdot 45) .43$ patients who were pre-treated with interferon for more than 1.5 years were removed from the learning sample because they were likely to represent positive selection of patients and because it is currently uncommon for a patient to be pre-treated with interferon for this long before starting TKI therapy.

For the 405 patients remaining in the learning sample (of whom 180 [44\%] had relapses), the association between deep molecular response duration before TKI discontinuation and probability of MMR at 6 months after TKI discontinuation remained significant (OR 1.13 [95\% CI 1.04 to $1 \cdot 23$ ]; $\mathrm{p}=0 \cdot 0032$ ). In combination with the intercept -0.38 (95\% CI -0.82 to 0.06$)$ estimated from the common logistic regression model, this OR translated into a yearly increase of about $3 \%$ in the probability of staying in MMR at 6 months over the observed range of deep molecular response durations. Table 3 shows the mean estimated and observed probabilities for 1-year intervals of deep molecular response duration.

Duration of imatinib treatment also remained significantly associated with probability of MMR at 6 months in the learning sample without patients pre-treated with interferon for more than 1.5 years (OR 1.14 [95\% CI 1.05-1.23]; $\mathrm{p}=0 \cdot 0010)$. However, because deep molecular response duration before TKI discontinuation was part of duration of TKI treatment, these parameters were strongly correlated. Because of this collinearity, the variable time of TKI treatment before deep molecular response was investigated. The effect of this variable on molecular relapse-free survival at 6 months was not significant (OR 1.04 [95\% CI 0.93-1.15]; $\mathrm{p}=0 \cdot 50$ ).

When considering all candidate variables for multivariable modelling, either deep molecular response duration before TKI discontinuation or duration of TKI treatment was significant. Because of their collinearity, only one of these variables could be kept in the model. The results of multivariable modelling with inclusion of deep molecular response duration before TKI discontinuation are shown in the appendix (p 9). Deep molecular response duration before TKI discontinuation and time of TKI treatment before deep molecular response showed a weak, negative correlation $(r=-0 \cdot 28)$. When time of TKI treatment before deep molecular response was added to deep molecular response duration before TKI discontinuation, only deep molecular response duration before TKI discontinuation remained significant $(\mathrm{p}=0.0009$ vs $\mathrm{p}=0.092$ for time of TKI treatment before deep molecular response). Looking at 1000 bootstrap samples, deep molecular response duration before TKI discontinuation was significant in 783 cases, and linear modelling was preferred over any fractional polynomial. The Hosmer-Lemeshow test did not indicate a poor goodness of fit $(\mathrm{p}=0 \cdot 40)$.

The validation sample consisted of 195 patients (of whom 73 [37\%] had relapses after 6 months). $30(15 \%)$ of these patients had received first-line nilotinib and $13(7 \%)$ had received dasatinib. Type of first-line TKI did not significantly affect molecular relapse-free survival at 6 months (appendix p 9). Similar to the learning population, treatment duration with TKI, deep molecular response duration, and interferon pretreatment were significantly associated with molecular relapse-free survival at 6 months after TKI discontinuation (appendix p 9). Molecular relapse-free survival at 6 months was $88 \%$ (95\% CI 68-97) in 24 patients who had been pre-treated with interferon for more than 1.5 years and $59 \%(51-67)$ in 171 patients pre-treated with interferon for 1.5 years or less (OR 4.85 [95\% CI $1 \cdot 39-16 \cdot 88] ; \mathrm{p}=0 \cdot 013)$.

After the 24 patients who had been pre-treated with interferon for more than 1.5 years were excluded from 


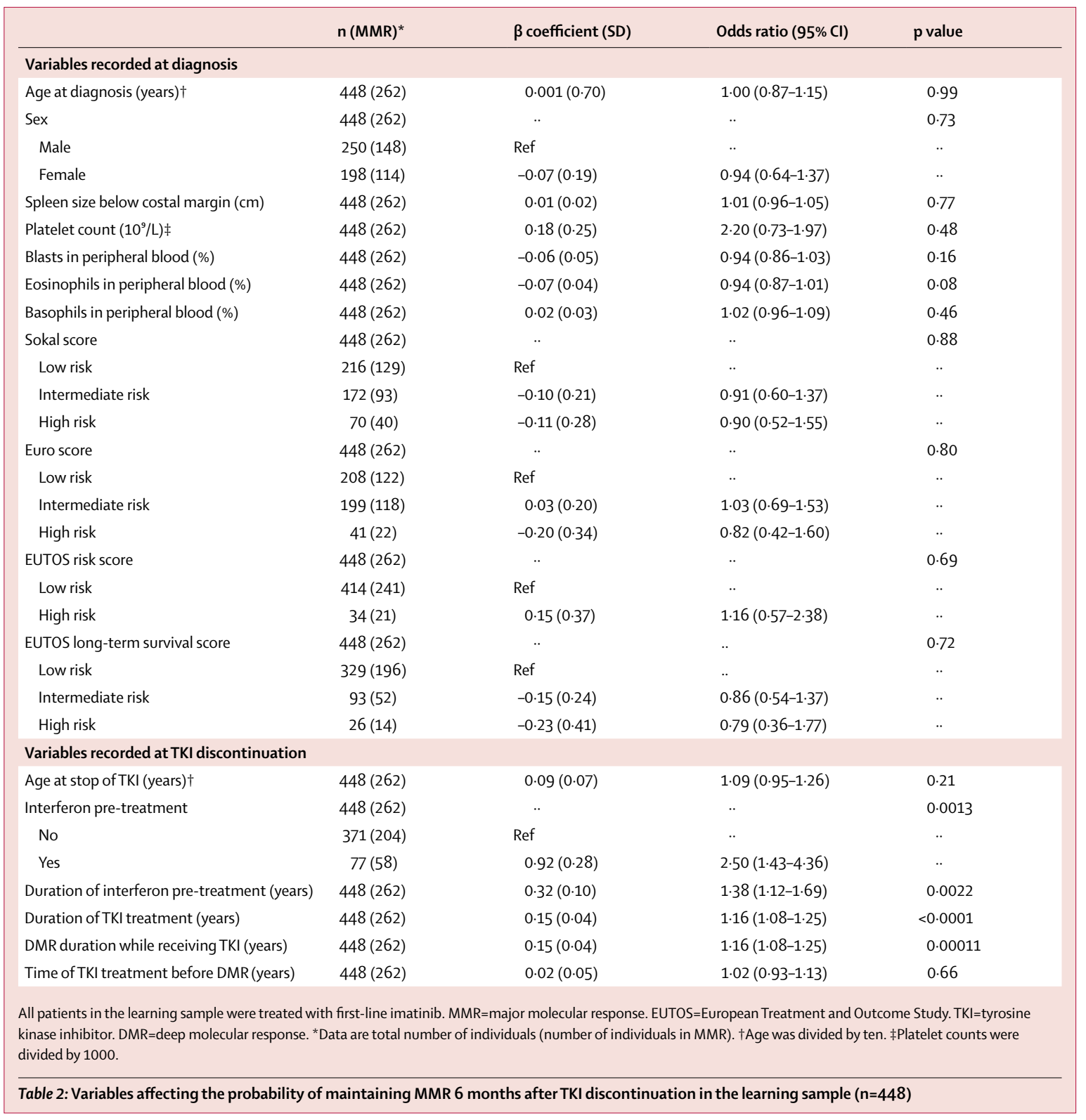

the analysis, deep molecular response duration before TKI discontinuation in the remaining 171 patients (of whom 70 [41\%] had relapses) had the same OR as in the learning sample, but the association was not significant (OR 1.13 [95\% CI 0 98-1.29]; p=0 084). Duration of TKI treatment was also not significant $(1.09$ [0.98-1.21]; $\mathrm{p}=0 \cdot 13)$.

Prognostic modelling in 405 patients in the learning sample led to two mutually exclusive univariate logistic models. Using the minimum $\mathrm{p}$ value approach for deep molecular response duration before TKI discontinuation, a cutoff of $3 \cdot 1$ years was observed $\left(p_{\text {adiusted }}=0 \cdot 0029\right)$, assuming that the smallest group contained at least $20 \%$ of patients. This cutoff was confirmed with bootstrap resampling: in 1000 bootstrap samples, the cutoff of $3 \cdot 1$ years was most frequently chosen (median $3 \cdot 3$ years [IQR 2.8-5.4]). For the 276 patients who had a deep molecular response while on treatment for more than 3.1 years, the probability of maintaining MMR at 6 months was 61\% (95\% CI 55-67; corresponding to the positive predictive value [PPV]), whereas it was $44 \%$ (35-53) for the 129 patients who had a deep molecular response for 3.1 years or less (OR 1.97 [95\% CI $1 \cdot 29-3 \cdot 00])$. The negative predictive value (NPV) was $56 \%$ (95\% CI 47-65), sensitivity was 75\% (68-80), and specificity was $40 \%(33-48)$.

For duration of imatinib treatment, $5 \cdot 8$ years $\left(\mathrm{p}_{\text {adiusted }}=0 \cdot 00090\right)$ was identified as the cutoff. For the 


\begin{tabular}{|c|c|c|c|}
\hline & $\mathrm{n} / \mathbf{N}^{*}$ & $\begin{array}{l}\text { Estimated } \\
\text { proportion } \\
\text { without } \\
\text { relapse }(\%) \dagger\end{array}$ & $\begin{array}{l}\text { Observed } \\
\text { proportion } \\
\text { without relapse } \ddagger\end{array}$ \\
\hline \multicolumn{4}{|c|}{ Learning sample } \\
\hline $1-2$ & $14 / 44$ & $45 \%$ & $32 \%(19-48)$ \\
\hline$>2$ to $\leq 3$ & $39 / 72$ & $49 \%$ & $54 \%(42-66)$ \\
\hline$>3$ to $\leq 4$ & $28 / 55$ & $51 \%$ & $51 \%(37-65)$ \\
\hline$>4$ to $\leq 5$ & $35 / 60$ & $55 \%$ & $58 \%(45-71)$ \\
\hline$>5$ to $\leq 6$ & $28 / 42$ & $58 \%$ & $67 \%(50-80)$ \\
\hline$>6$ to $\leq 7$ & $25 / 48$ & $61 \%$ & $52 \%(37-67)$ \\
\hline$>7$ to $\leq 8$ & $27 / 34$ & $64 \%$ & $79 \%(62-91)$ \\
\hline$>8$ to $\leq 9$ & $10 / 21$ & $66 \%$ & $48 \%(26-70)$ \\
\hline$>9$ & $19 / 29$ & $70 \%$ & $66 \%(46-82)$ \\
\hline \multicolumn{4}{|c|}{ Validation sample } \\
\hline $1-2$ & $13 / 28$ &.. & $46 \%(28-66)$ \\
\hline$>2$ to $\leq 3$ & $17 / 31$ &.. & $55 \%(36-73)$ \\
\hline$>3$ to $\leq 4$ & $22 / 37$ &.. & $59 \%(42-75)$ \\
\hline$>4$ to $\leq 5$ & $11 / 17$ &.. & $65 \%(38-86)$ \\
\hline$>5$ to $\leq 6$ & $14 / 20$ &.. & $70 \%(46-88)$ \\
\hline$>6$ to $\leq 7$ & $9 / 15$ &.. & $60 \%(32-84)$ \\
\hline$>7$ & $15 / 23$ &.. & $65 \%(43-84)$ \\
\hline \multicolumn{4}{|c|}{$\begin{array}{l}\text { MMR=major molecular response. TKI=tyrosine kinase inhibitor. }{ }^{*} \text { Data are number } \\
\text { of patients without relapse/total number of patients. †Mean proportion } \\
\text { estimated for each individual deep molecular response duration from the logistic } \\
\text { regression model. } \neq \text { Data are } \%(95 \% \mathrm{Cl}) \text {. }\end{array}$} \\
\hline \multicolumn{4}{|c|}{$\begin{array}{l}\text { Table 3: Probability of maintaining MMR } 6 \text { months after TKI } \\
\text { discontinuation, by duration of deep molecular response (years) before } \\
\text { imatinib discontinuation }\end{array}$} \\
\hline
\end{tabular}

267 patients who were treated with imatinib for more than 5.8 years, molecular relapse-free survival at 6 months was $63 \%$ (95\% CI 57-69; corresponding to the PPV), whereas it was $41 \%(33-50)$ for the 138 patients who were treated for up to 5.8 years (OR 2.41 [95\% CI 1.58-3.67]). The NPV was 59\% (95\% CI 50-67), sensitivity was $75 \%$ (95\% CI 69-80), and specificity was $45 \%$ (95\% CI 38-53).

We tested the cutoffs for deep molecular response duration while receiving imatinib and duration of imatinib treatment in the 171 patients in the validation sample. For the 106 patients who had a deep molecular response for more than 3.1 years while receiving imatinib, molecular relapse-free survival at 6 months was $62 \%$ (95\% CI 52-72; corresponding to the PPV), whereas it was $54 \%(41-66)$ for the 65 patients who had a deep molecular response of $3 \cdot 1$ years or less (OR 1.41 [95\% CI 0.76-2.65]; $\mathrm{p}=0 \cdot 28)$. Thus, the NPV was $46 \%$ (95\% CI $34-59)$, sensitivity was 65\% (95\% CI 55-75), and specificity was $43 \%$ (95\% CI 31-55).

For the 89 patients who were treated with imatinib for more than 5.8 years, molecular relapse-free survival at 6 months was $63 \%$ (95\% CI 52-73; corresponding to the PPV), whereas it was 55\% (95\% CI 43-66) for the 82 patients treated with imatinib for 5.8 years or less (OR 1.40 [95\% CI $0 \cdot 76-2 \cdot 57] ; p=0 \cdot 29$ ). Thus, the NPV was $45 \%$ (95\% CI 34-57), sensitivity was 55\% (95\% CI 45-65), and specificity was 53\% (95\% CI 41-65).

596 patients with complete information about first-line imatinib treatment were considered assessable in the cost analysis. 279 patients restarted treatment after a median of 4 months (range 1-36), and 317 patients were still treatment-free at the last analysis, with a median time off treatment of 26 months (1-39). The total time off treatment was 1712 months in patients who were retreated and 8092 months in patients who stopped treatment. The mean cost of imatinib per month was $€ 2262$. Considering only the drug price, the total savings were $€ 22$ million. These savings were reduced by supplementary investigations (eg, clinical visits, PCR analysis, examinations). We calculated that the median cost of a PCR test was €300. Within the first year, six additional tests per patient were necessary, resulting in costs of about $€ 1.5$ million. Because the cost of generic imatinib varies between countries, savings with generic imatinib were difficult to calculate, but continued to increase each month for the 317 patients who were off treatment at the time of writing.

No losses of complete haematological remission or progression due to accelerated-phase chronic myeloid leukaemia or blast phase were reported. 83 (11\%) of 755 patients were no longer in complete cytogenetic remission after loss of MMR, including 11 cases confirmed with cytogenetic evaluation. $13(2 \%)$ patients restarted TKI without loss of MMR because of patient's wish or treating physician's decision. After restarting treatment, $321(86 \%)$ of 373 patients had an MMR, of whom 302 (81\%) were in deep molecular response. Median time to regaining MMR after resuming TKI therapy was 2.8 months $(95 \%$ CI $2 \cdot 7-2 \cdot 9)$ and to regaining deep molecular response was 3.7 months $(3 \cdot 5-4 \cdot 2)$. Because half of the patients had a follow-up of less than 1 year after restarting treatment (median 11 months [IQR 8-15]), these results are preliminary.

Ten patients died for reasons unrelated to chronic myeloid leukaemia, including four who died while in MMR (due to myocardial infarction, lung cancer, renal cancer, and heart failure) and six (2\%) who died in chronic-phase chronic myeloid leukaemia after loss of MMR and re-initiation of TKI therapy (due to myocardial infarction, cerebral infarction, pancreatic cancer, septicaemia, pneumonia, and heart failure). The remaining 378 patients were still alive and in MMR, including 11 who had restarted TKI therapy and 367 who were not on treatment.

Adverse events were prospectively assessed (table 4). TKI withdrawal syndrome was noticed in 233 (31\%) of 758 patients, which was in line with a previous report. ${ }^{21}$ Mostly transient, TKI withdrawal syndrome consisted of musculoskeletal or joint pain. 224 (30\%) of 758 patients reported a grade 1-2 TKI withdrawal syndrome and nine (1\%) patients reported a grade 3 TKI withdrawal syndrome (table 4) during the treatment period. 


\section{Discussion}

We found that cessation of TKI therapy in patients with chronic myeloid leukaemia in deep molecular response resulted in a molecular relapse-free survival of 61\% (95\% CI 57-64) and a molecular relapse-free and treatment-free survival of $60 \%(56-63)$ at 6 months. Stopping TKI therapy appears feasible and safe when applying predefined criteria and decentralised, but standardised, molecular monitoring.

This study outlines important preconditions that can be used as guidance for defining stopping criteria. To our knowledge, this study is the first to show that more exact criteria for successful discontinuation of treatment could be defined with prognostic modelling to predict molecular relapse-free survival at 6 months in a large cohort of patients who discontinued imatinib. The large cohort of patients in the trial and the use of less stringent stopping criteria than used previously-11,22,23 allowed the importance of duration of molecular remission to be validated before TKI discontinuation was attempted.

Although our criteria for TKI discontinuation were less stringent $\left(4-\log _{10}\right.$ reduction or lower) than those in the STIM1 study (patients with negative PCR results), ${ }^{7}$ the results of the two studies were similar, with most molecular recurrences occurring within the first 6 months after TKI discontinuation. Similarly, the criterion for retreatment in this study (loss of MMR at any timepoint) was less stringent than that in STIM1 (positivity for $B C R-A B L 1$ transcript in two consecutive rtPCR assessments, with a $1-\log _{10}$ increase in the second assessment relative to the first, or loss of MMR in one assessment). ${ }^{\text {? }}$ However, we confirmed and validated that loss of MMR is a safe criterion for TKI retreatment, as was previously shown in the A-STIM study. ${ }^{8}$ This criterion increases the patient population eligible for treatment discontinuation.

Because results from previous studies ${ }^{7,910,22,24}$ showed that $80 \%$ of molecular relapses occurred in the first 6 months, we decided to assess factors affecting probability of maintaining MMR at 6 months. We found that duration of deep molecular response before TKI cessation was the most important factor affecting probability of remaining in MMR or better at 6 months after treatment discontinuation. Additionally, we found a roughly linear increase in the probability of molecular relapse-free survival per additional year in deep molecular response for patients treated with first-line imatinib.

The two components of total treatment time, time before deep molecular response and time after deep molecular response, are not independent of each other. Our results show the importance of deep molecular response duration in predicting molecular relapse-free survival. For duration of deep molecular response, both the learning and validation cohorts had the same OR of $1 \cdot 13$, with a wider confidence interval in the smaller validation sample $(95 \%$ CI $0.98-1.29$ vs 1.04-1.23). Duration of deep molecular response was more important than total treatment time because it was

\begin{tabular}{|c|c|c|c|c|}
\hline & Grade 1-2 & Grade 3 & Grade 4 & Grade 5 \\
\hline \multicolumn{5}{|l|}{ Cardiovascular } \\
\hline $\begin{array}{l}\text { Coronary disease (angina, myocardial } \\
\text { infarction, ACS) }\end{array}$ & .. & $2(<1 \%)$ & $4(<1 \%)$ & $2(<1 \%)$ \\
\hline Heart failure & .. & $2(<1 \%)$ & .. & $2(<1 \%)$ \\
\hline Hypertension & .. & $3(<1 \%)$ & $1(<1 \%)$ &.. \\
\hline Stroke or transient ischaemic attack & .. & $2(<1 \%)$ & $1(<1 \%)$ & $1(<1 \%)$ \\
\hline Arrhythmia & .. & $1(<1 \%)$ & .. & .. \\
\hline Deep vein thrombosis or thrombophlebitis & .. & $2(<1 \%)$ & .. & .. \\
\hline \multicolumn{5}{|l|}{ Respiratory } \\
\hline Cough or dyspnoea & .. & $3(<1 \%)$ &.. & .. \\
\hline Pneumonia & .. & $2(<1 \%)$ & .. & $1(<1 \%)$ \\
\hline Oedema of throat & .. & $1(<1 \%)$ &.. & .. \\
\hline \multicolumn{5}{|l|}{ Renal and urinary tract } \\
\hline Pyelonephritis & .. & $1(<1 \%)$ & .. & .. \\
\hline Urinary retention & .. & $1(<1 \%)$ & .. & .. \\
\hline \multicolumn{5}{|l|}{ Hepatic and gastrointestinal tract } \\
\hline Hepatic abscess & .. & $1(<1 \%)$ &.. & .. \\
\hline Diverticulitis & .. & $1(<1 \%)$ & .. & .. \\
\hline Choledocholithiasis with biliary fistula & .. & $1(<1 \%)$ &.. & .. \\
\hline Intestinal disorder & .. & $1(<1 \%)$ & .. & .. \\
\hline \multicolumn{5}{|l|}{ Infectious } \\
\hline Septicaemia & .. & .. &.. & $1(<1 \%)$ \\
\hline Herpes zoster & .. & $1(<1 \%)$ &.. &. \\
\hline Gluteal abscess & .. & $1(<1 \%)$ & .. & .. \\
\hline \multicolumn{5}{|l|}{ Vision } \\
\hline Chorioretinitis & .. & $1(<1 \%)$ & .. & .. \\
\hline Corneal ulcer & .. & $1(<1 \%)$ &.. &. \\
\hline Diplopia & .. & $1(<1 \%)$ &.. & .. \\
\hline Cataract & .. & $1(<1 \%)$ & .. & .. \\
\hline \multicolumn{5}{|l|}{ CNS or psychiatric } \\
\hline Depression & .. & $1(<1 \%)$ &.. & .. \\
\hline Memory impairment & .. & $1(<1 \%)$ &.. & .. \\
\hline Vestibular neuropathy & .. & $1(<1 \%)$ & .. & .. \\
\hline \multicolumn{5}{|l|}{ Musculoskeletal pain* } \\
\hline Total & $263(35 \%)$ & $11(1 \%)$ & .. & .. \\
\hline During treatment-free period & $224(30 \%)$ & $9(1 \%)$ & .. & .. \\
\hline \multicolumn{5}{|l|}{ Other } \\
\hline Hypothyroidism & .. & $1(<1 \%)$ & .. & .. \\
\hline Spinal stenosis & .. & $1(<1 \%)$ &.. & .. \\
\hline Osteoporotic fracture & .. & $1(<1 \%)$ &.. &. \\
\hline Alopecia & .. & $1(<1 \%)$ & .. & .. \\
\hline $\begin{array}{l}\text { Data are number of events (\%). The denominato } \\
\text { were reported in more than } 10 \% \text { of patients in th } \\
\text { number of patients because patients might have } \\
\text { musculoskeletal pain, bone or joint pain, arthral } \\
\text { pain, arthromyalgia, pain in both arms, pain in le }\end{array}$ & $\begin{array}{l}\text { is } 758 \text {. All grac } \\
\text { ee study are sho } \\
\text { experienced se } \\
\text { gia, myalgia, joi } \\
\text { egs, and related }\end{array}$ & $\begin{array}{l}3,4 \text { and } 5 \text { ad } \\
\text { The total nu } \\
\text { ral events. AC } \\
\text { stiffness, lum } \\
\text { ms. }\end{array}$ & $\begin{array}{l}\text { ovents and } \\
\text { of events } n \\
\text { te coronary } \\
\text { articular } p\end{array}$ & $\begin{array}{l}\text { de } 1-2 \text { events tha } \\
\text { exceed the } \\
\text { rome. *Includes } \\
\text { uscular pain, neck }\end{array}$ \\
\hline
\end{tabular}

significant on its own, whereas the first component of total treatment time, time before deep molecular response, was not significant. However, the two parts were negatively correlated, and the $\mathrm{p}$ value of time before deep molecular response was 0.092 in the multivariable analysis. The fact that the date of the first observation of 
deep molecular response was retrieved retrospectively and, therefore, might not always be exact is a limitation of this study. Because of these results, together with the surmised lack of exactness and for patient safety, total treatment time was also investigated and discussed.

The best cutoff for deep molecular response duration was defined as $3 \cdot 1$ years in the learning sample. Also significant, but of less importance, was the duration of imatinib therapy: more than 5.8 years of treatment before discontinuation increased the probability of molecular relapse-free survival at 6 months. Although these cutoffs could not be validated in the independent cohort, physicians should consider that the likelihood of treatment discontinuation succeeding might decrease or increase with otherwise shorter or longer treatment durations. Additionally, late relapses were not evaluated at this timepoint (planned after 3 years' follow-up). Prospective evaluations in newly diagnosed patients, particularly those treated with second-generation TKI therapy, is needed for further insight.,10

The STIM1 study found that duration of treatment was a significant predictive factor of molecular relapse-free survival. Treatment duration of at least 4.5 years was associated with a reduced risk of molecular recurrence; however, half of the patients in STIM1 had previously been treated with interferon, which was found to be an important factor in this study. By contrast with the STIM1 study, we found that Sokal and EUTOS risk scores were not predictive of maintaining MMR.

The biological mechanisms of relapse after TKI discontinuation are not clearly understood, but they are an important and central part of chronic myeloid leukaemia research. ${ }^{25}$ An increased proportion of mature natural killer cells was associated with successful imatinib discontinuation in chronic myeloid leukaemia. ${ }^{26,27}$ Furthermore, expression of the CTLA-4 ligand, CD86, on plasmacytoid dendritic cells was shown to predict relapse risk after TKI discontinuation. ${ }^{28}$ Other mechanisms, such as aspects of stem cell biology, might also affect risk of relapse.

Because the life expectancy of patients with chronic myeloid leukaemia treated with TKI is now close to that of the general population, research on improving the management of this chronic disease is now focused on quality of life and economic aspects..$^{29}$ TKI discontinuation has a large economic impact, with an estimated saving of $€ 22$ million alone for the participants in this study. The safety of TKI discontinuation has been an important issue. If discontinuation is done according to the procedures used in this trial, particularly with standardised molecular monitoring of the major type of the $B C R-A B L 1$ transcript, it is safe and practicable. Rapid reporting of PCR results ensures prompt TKI resumption upon molecular recurrence of the disease. Patients and physicians should also be aware of transient TKI withdrawal syndrome. ${ }^{21,30}$

In summary, a discontinuation attempt could be suggested for patients fulfilling the criteria of this study because no safety issues were reported. To boost the likelihood of remaining recurrence-free, durations of deep molecular response and TKI treatment are important to take into account. For both parameters, a continuous, per-year increase in the probability of maintaining MMR at 6 months was observed in the learning cohort, with deep molecular response duration being more important than duration of treatment. These continuous increases were also observed in the validation cohort. Estimates of the probability of molecular relapsefree survival with each additional year in deep molecular response before stopping imatinib could be used as guidance for physicians making decisions about individual patients. Physicians should weigh the sideeffects and costs of prolonged treatment against the probability of staying in MMR.

\section{Contributors}

SS, F-XM, JG, MP, and JR developed the study design and wrote the Article. FXG, HH-H, AA, JJWMJ, JM, PK, PP, UÖ-S, JM-L, HV, and SM coordinated the study in their countries. HE, VK, KMP, MCM, and AF did the PCR analyses in the standardised national laboratories. MGB, PR, W-KH, and AH led recruitment, treatment, and data collection in their study centres. All authors had full access to all the data in the study, participated in the analysis and interpretation of the data, contributed to the writing and revision of all drafts, approved the final version, and agreed to submit the paper for publication.

\section{Declaration of interests}

SS reports grants from ELN Foundation, during the conduct of the study, and grants and personal fees from Novartis and Bristol-Myers Squibb (BMS), and personal fees from Pfizer and Incyte, outside the submitted work. JR reports personal fees from Novartis, and grants and personal fees from Pfizer, outside the submitted work. HH-H reports grants from BMS grants and personal fees from Pfizer, and personal fees from Janssen, outside the submitted work. AA reports personal fees from Novartis, BMS Celgene, Alexion, and Servier, outside the submitted work. JJWMJ reports grants, personal fees, and honoraria from Novartis; grants and personal fees from BMS; personal fees from Pfizer; and honoraria from Incyte, outside the submitted work. JM reports grants from Novartis and BMS, outside the submitted work. KMP reports personal fees from Incyte and Novartis, and grants and personal fees from BMS, outside the submitted work. MCM reports personal fees from Novartis, BMS, Pfizer, and Incyte, and Institute for Hematology and Oncology, outside the submitted work. SM reports grants and personal fees from BMS and Novartis, grants from Pfizer, and personal fees from Celgene, outside the submitted work. PR reports personal fees from Amgen, grants and personal fees from Incyte, and grants from Pfizer and BMS, outside the submitted work. AH reports grants, personal fees, and non-financial support from Novartis and BMS; grants from Incyte; and grants and personal fees from Ariad and Pfizer, outside the submitted work. MP reports personal fees from Novartis and BMS, outside the submitted work. F-XM reports grants from National Cancer Institute France, during the conduct of the study. F-XM also reports grants, personal fees, and honoraria from Novartis, and personal fees from BMS, Pfizer, and Incyte, outside the submitted work. All other authors declare no competing interests.

\section{Acknowledgments}

Heidelberg University was the formal sponsor of the study on behalf of ELN. For the French part of the trial, financial support was given partially by the National Cancer Institute. We thank and pay tribute to our deceased colleagues John Goldman and Josy Reiffers for their support at the start of the study. We also thank Alois Gratwohl (Klinik für Hämatologie, Universitätsspital Basel, Basel, Switzerland) and all members of the ELN for their support and encouragement. We thank Rüdiger Hehlmann (III Medizinische Klinik, Medizinische Fakultät Mannheim Universität, Heidelberg, Germany) for continued support and for making the infrastructure of the CML Study Centre Mannheim available to us. We also thank Severine Clerjaud and Violaine Goyeau (both affiliated with Inserm, 
Centre d'Investigation Clinique 1402, Centre Hospitalier Universitaire de Poitiers, Poitiers, France) who managed and did quality control for the European EURO-SKI database. We thank Gabriele Bartsch (Department of Haematology and Oncology, University Hospital Mannheim, Heidelberg University, Mannheim, Germany) for logistical support, Arthur Gil (Institut für Medizinische Informationsverarbeitung, Biometrie und Epidemiologie, Ludwig-Maximilians-Universität, Munich, German) for data preparation, and Kathrin Halfter (Institut für Medizinische Informationsverarbeitung, Biometrie und Epidemiologie, LudwigMaximilians-Universität, Munich, Germany) for carefully reading the manuscript. Finally, we thank the patients for their participation and the involved staff on study sites.

\section{References}

1 Heisterkamp N, Stephenson JR, Groffen J, et al. Localization of the c-ab1 oncogene adjacent to a translocation break point in chronic myelocytic leukaemia. Nature 1983; 306: 239-42.

2 Druker BJ, Guilhot F, O’Brien SG, et al. Five-year follow-up of patients receiving imatinib for chronic myeloid leukemia. $N$ Engl J Med 2006; 355: 2408-17.

3 Pfirrmann M, Baccarani M, Saussele S, et al. Prognosis of long-term survival considering disease-specific death in patients with chronic myeloid leukemia. Leukemia 2016; 30: 48-56.

4 Bower H, Bjorkholm M, Dickman PW, Hoglund M, Lambert PC, Andersson TM. Life expectancy of patients with chronic myeloid leukemia approaches the life expectancy of the general population. J Clin Oncol 2016; 34: 2851-57.

5 Hochhaus A, Larson RA, Guilhot F, et al. Long-term outcomes of imatinib treatment for chronic myeloid leukemia. N Engl J Med 2017; 376: 917-27.

6 Hehlmann R, Lauseker M, Saußele S, et al. Assessment of imatinib as first-line treatment of chronic myeloid leukemia: 10-year survival results of the randomized CML study IV and impact of non-CML determinants. Leukemia 2017; 31: 2398-406.

7 Etienne G, Guilhot J, Rea D, et al. Long-term follow-up of the French Stop Imatinib (STIM1) study in patients with chronic myeloid leukemia. J Clin Oncol 2017; 35: 298-305.

8 Rousselot P, Charbonnier A, Cony-Makhoul P, et al. Loss of major molecular response as a trigger for restarting tyrosine kinase inhibitor therapy in patients with chronic-phase chronic myelogenous leukemia who have stopped imatinib after durable undetectable disease. J Clin Oncol 2014; 32: 424-30.

9 Rea D, Nicolini FE, Tulliez M, et al. Discontinuation of dasatinib or nilotinib in chronic myeloid leukemia: interim analysis of the STOP 2G-TKI study. Blood 2017; 129: 846-54.

10 Hochhaus A, Masszi T, Giles FJ, et al. Treatment-free remission following frontline nilotinib in patients with chronic myeloid leukemia in chronic phase: results from the ENESTfreedom study. Leukemia 2017; 31: 1525-31.

11 Saussele S, Richter J, Hochhaus A, Mahon FX. The concept of treatment-free remission in chronic myeloid leukemia. Leukemia 2016; 30: 1638-47.

12 Cross NCP, White HE, Colomer D, et al. Laboratory recommendations for scoring deep molecular responses following treatment for chronic myeloid leukemia. Leukemia 2015; 29: 999-1003.

13 Baccarani M, Deininger MW, Rosti G, et al. European LeukemiaNet recommendations for the management of chronic myeloid leukemia: 2013. Blood 2013; 122: 872-84.

14 Müller MC, Erben P, Saglio G, et al. Harmonization of $B C R-A B L$ mRNA quantification using a uniform multifunctional control plasmid in 37 international laboratories. Leukemia 2008; 22: 96-102.
15 Mahon FX, Rea D, Guilhot J, et al. Discontinuation of imatinib in patients with chronic myeloid leukaemia who have maintained complete molecular remission for at least 2 years: the prospective, multicentre Stop Imatinib (STIM) trial. Lancet Oncol 2010; 11: 1029-35.

16 Putter H, Fiocco M, Geskus RB. Tutorial in biostatistics: competing risks and multi-state models. Stat Med 2007; 26: 2389-430.

17 Royston P, Sauerbrei W. Multivariable model-building: a pragmatic approach to regression analysis based on fractional polynomials for modelling continuous variables. Chichester: John Wiley \& Sons, 2008.

18 Davison AC, Hinkley DV. Bootstrap methods and their application. Cambridge: Cambridge University Press, 1997.

19 Hosmer DW, Lemeshow S. Applied logistic regression. New York, NY: John Wiley \& Sons, 2005.

20 Altman DG, Lausen B, Sauerbrei W, Schumacher M. Dangers of using "optimal" cutpoints in the evaluation of prognostic factors. J Natl Cancer Inst 1994; 86: 829-35.

21 Richter J, Soderlund S, Lubking A, et al. Musculoskeletal pain in patients with chronic myeloid leukemia after discontinuation of imatinib: a tyrosine kinase inhibitor withdrawal syndrome? J Clin Oncol 2014; 32: 2821-23.

22 Imagawa J, Tanaka H, Okada M, et al. Discontinuation of dasatinib in patients with chronic myeloid leukaemia who have maintained deep molecular response for longer than 1 year (DADI trial): a multicentre phase 2 trial. Lancet Haematol 2015; 2: e528-35.

23 Clark RE, Polydoros F, Apperley JF, et al. De-escalation of tyrosine kinase inhibitor dose in patients with chronic myeloid leukaemia with stable major molecular response (DESTINY): an interim analysis of a non-randomised, phase 2 trial. Lancet Haematol 2017; 4: e310-16.

24 Campiotti L, Suter MB, Guasti L, et al. Imatinib discontinuation in chronic myeloid leukaemia patients with undetectable BCR-ABL transcript level: a systematic review and a meta-analysis. Eur J Cancer 2017; 77: 48-56.

25 Hamilton A, Helgason GV, Schemionek M, et al. Chronic myeloid leukemia stem cells are not dependent on Bcr-Abl kinase activity for their survival. Blood 2012; 119: 1501-10.

26 Ilander M, Olsson-Stromberg U, Schlums H, et al. Increased proportion of mature NK cells is associated with successful imatinib discontinuation in chronic myeloid leukemia. Leukemia 2016; 31: 1108-16.

27 Rea D, Henry G, Khaznadar Z, et al. Natural killer-cell counts are associated with molecular relapse-free survival after imatinib discontinuation in chronic myeloid leukemia: the IMMUNOSTIM study. Haematologica 2017; 102: 1368-77.

28 Schütz C, Inselmann S, Sausslele S, et al. Expression of the CTLA-4 ligand CD86 on plasmacytoid dendritic cells (pDC) predicts risk of disease recurrence after treatment discontinuation in CML. Leukemia 2017; 31: 829-36.

29 Experts in Chronic Myeloid Leukemia. The price of drugs for chronic myeloid leukemia (CML) is a reflection of the unsustainable prices of cancer drugs: from the perspective of a large group of CML experts. Blood 2013; 121: 4439-42.

30 Lee S-E, Choi SY, Song H-Y, et al. Imatinib withdrawal syndrome and longer duration of imatinib have a close association with a lower molecular relapse after treatment discontinuation: the KID study. Haematologica 2016; 101: 717-23. 